\section{Challenges in morphological diagnosis of tuberculosis}

10.52739/bio-up.1.2021.28-35

Petro V. Kuzyk', Andriy Yu. Horoshchak², Bogdan Ya. Serbin², Rahaina Musa-Bador', Uchenna Uchie-Okoro ${ }^{2}$

\section{ABSTRACT}



$B$ is the seventh leading cause of death worldwide and third leading cause of death among women in the 15-44 age group [1]. However, in 2010, the number of multidrug-resistant tuberculosis (MDR-TB) cases rose to 650,000 worldwide, with more than 150,000 deaths. All of the well-known widely available methods such as chest $\mathrm{X}$-ray, culture in liquid and solid media should be accompanied with the modern and more accurate methods such as chest CT and molecular diagnostic tests which proved the necessity of usage with high sensitivity and accuracy, especially in smearnegative pulmonary TB patients and patients with the multi drug resistant forms.

Bogomolets National Medical University, Department of Pathological Anatomy \#2, Kyiv, Ukraine

Dathological Anatomy, Histology and Forensic Medicine, Kyiv, Ukraine

\section{INTRODUCTION}

Tuberculosis (TB) is a curable and preventable disease caused by infection with mycobacteria from the Mycobacterium tuberculosis complex. M. tuberculosis can cause disease in any organ of the body, but TB infection of the lung is significant [2] because it spreads via respiratory droplets; this makes it a major public health burden (despite the availability of definitive treatment for more than five decades). A majority of death due to tuberculosis occur majority of death due to tuberculosis occur as a result of pulmonary disease [3]. Nowadays, TB become a huge global issue for the both developing and developed countries. Due to spreading of multi drug-resistant forms and variety of symptoms it remains to be a huge burden on global health care system $[4,5]$. The variety of TB forms and difficulties in differential diagnostics results in mistakes among clinicians in treatment of TB patients $[6,7]$. All of those issues results in developing of the new diagnostic methods and tests which already implemented into some countries' health care systems for improvement of $T B$ diagnosis [8]. However, those methods should receive availability for all health care systems globally which will provide higher rates of correct diagnosis of the TB.

\section{TRANSMISSION OF TB}

TB in humans is transmitted through respiratory droplets from person to person usually by coughing but also by sneezing and talking. The size of the droplet is critical for infection because transmission most often ccurs with infected droplets of respirable size (approximatel size (approxim and retained in the alveoli of a susceptible host. Approximately six mycobacteria are carried to the level of the alveolus by each
respirable droplet [10] Mycobacteria enter alveolar macrophages of the host by active alveolar macrophages of the host by active where the internalized mycobacteria then alter the macrophage phagosome to make that environment less hostile and to prevent mycobacterial killing. Mycobacteria then replicate intracellularly which eventually results in macrophage death, cell rupture and the release of new mycobacteria that set up a focus of pulmonary infection in the alveolus. Approximately 5-200 bacilli are required to withstand the macrophagic defense mechanism before establishing infection within the lungs.

\section{RISK FACTORS FOR INFECTION}

Impaired Host Immunity

Certain lifestyles predispose exposed people to tuberculosis infection. These include factors that reduce host immunity. Malnutrition also results in impaired cellular responses to mycobacterial antigens as well as educed immunoglobulins and innate immune factors [11]. Excessive alcohol consumption has been shown to acutely impair alveolar macrophage function as well as producing a all in immunoglobulin when end-stage liver disease occurs. Recreational drug abusers are also at high risk of TB through a complicated host-environmental interface [12].

\section{Host Genetics}

Genetics has been shown to have an important role in host defense and susceptibility to TB. There is a critical role for interleukin-12 (IL-12) interferon gamma (IFN- $\gamma$ ) and tumor necrosis factor (TNF) in the formation and function of the tuberculous granuloma. Recent studies have shown that variants of the cytokineinducible SRC homology 2 domain protein Chromogenic in Situ Hybridization (CISH) allele (necessary for IL-12 signaling) have been associated with an increased susceptibility to TB $[13,14]$. Families with polymorphisms of L-12, IFN- $\gamma$, TNF48 or their receptors have excessive susceptibility to mycobacteria infections (usually detected by excessive susceptibility to BCG disease).

Immunosuppressive and Immunomodulatory Drugs

mmune modulation is a rapidly expanding arm of the therapeutic armamentarium in developed countries. In particular, the selective mmune deficiency induced by TNF antagonist medication (Infliximab) used for rheumatoid disease and seronegative arthropathies has resulted in exquisite susceptibility to uberculosis in treated patients [15]. This has ed to guidelines suggesting that patients contemplating this treatment should be fully evaluated for latent tuberculosis and $\underset{\mathcal{N}}{\mathcal{N}}$ 
treated with six months of anti-tuberculous AFB smear microscopy and culture chemotherapy in case of doubt [16].

\section{Close Contact with Individuals with Active Tuberculosis}

The single most important risk factor for acquisition of tuberculosis is contact with a person who has active tuberculosis, particularly sputum smear positive (open) pulmonary tuberculosis. The most affected groups are those with recent travel to highly endemic areas or those who have had contact with people who have recently arrived from endemic areas.

\section{DIAGNOSTIC METHODS}

\section{Radiological method}

The diagnosis of TB starts with detection of symptoms which appeared. Such symptoms as subfebrile temperature for over 2 weeks and coughing which lasts for the same period or more as well as fatigue and weight period or more as well as fatigue and weight loss altogether are valuable reasons to
assume the appearance of TB [17]. The basic radiology method of early diagnosis for radiology method of early diagnosis for the lung TB is a chest X-ray. Although this
method is not specific for the TB diagnosis method is not specific for the TB diagnosis it allows to monitor the current condition of complications. Due to the fact that X-ray is not specific diagnostic procedure it has to be followed up with sputum testing. The chest $\mathrm{CT}$ is another one radiological method for the TB diagnosis. It could provide much more detailed information about the all the more detailed information about the all the lesions as well as complications which could [18]. On the CT scans TB could be visualized with such signs as pulmonary cavitation, with such signs as pulmonary cavitation,
branching opacities or "tree-in-bud" pattern which could be described as connection of

30 small centrilobular nodules of soft tissue

attenuation with multiple branching linear

structures. Though "tree-in-bud" symptom

could be detected in various condition except the lung TB but this symptom in association with cavitation or nodular opacities and with the classic TB symptoms could be evaluated as a confirmation of the pulmonary TB diagnosis [19]
The most frequent and useful test for the pulmonary $\mathrm{Tb}$ diagnosis is sputum test. One of its biggest advantages is availability - it could be provided in most primary health care laboratories [20]. It is highly specific for the Pulmonary TB and the rates of identification with the using of two consecutive sputum specimens are mostly very high (up to $95-$ $98 \%)[21,22]$ among smear-positive patients Nowadays, WHO recomositive TB potients. Nowadays, WHO recommends two consecutive sputum specimens testing among While with high rates of the TB spreading. While the resources are limited the ZiehNeelsen stained is the most common method. It is a highly specific method in TB diagnosis, however the levels of sensitivity could be very variable $(20-80 \%)$. The using of conventional fluorescence microscopy has much highe sensitivity in comparison with Ziehl-Neelsen microscopy although the limitations for using of this method are the requirement for the special expensive equipment [23]

However, there are some other challenges during the TB diagnostics. One of the is the diagnostic of drug-resistant strains which cannot be identified with the using of the chniques which were described above. In such case the drug susceptibility testing (DST) should be used. For this method the liquid media is the best choice for culturing of the TB bacilli because it allows to get the fina results in 10-14 days in comparison to 4-8 weeks which you will need in case of using other media. Moreover, the sensitivity of this method is approximately $10 \%$ higher in comparison with solid media. As a result liquid media could contribute significantly to the TB a faster and more accurate way of diagnostics. The only pros for this method are higher costs for its conduction and also its technical complexity is much higher in comparison with the using of solid media.

\section{Molecular methods}

The detection of TB could also be performed with the using of Nucleic acid amplification tests (NAATs). In comparison with the AFB smear microscopy this test is very useful in AFB smear-negative, culture-positive cases (it is up to $80 \%$ more sensitive). Moreover it could provide much faster detection of
M. tuberculosis in comparison with culturing methods in patients with pulmonary TB $[24,25,26]$. However this type of testing should not be used on a routine basis for patients with low clinical suspicion of $\mathrm{TB}$, because the PPV of NAAT is less than $50 \%$ in such cases [27].

\section{Line probe assay}

In order to provide much faster detection drugresistant cultures the Line probe assay method was developed. It allows to detect specific gene markers associated with rifampicin resistance alone or in combination with isoniazid $[28,29]$. It is conducted with the using of polymerase chain reaction (PCR) amplification of specific regions of the gene which are responsible for the drug-resistance. The using of this method showed very high sensitivity (more than $97 \%$ ) and specificity ( $\geqslant 99 \%$ ) for detecting rifampicin resistance alone or in combination with isoniazid (sensitivity $\geqslant 90 \%$; specificity $\geqslant 99 \%$ ), in M. tuberculosis isolates and in smearpositive sputum specimen [30]. Despite the very good results which were listed above this test should still be used with the culturing of $M$. tuberculosis and further DST because of the requirement of second-line anti-TB drugs using.

\section{Xpert MTB/RIF}

One of the most recent methods in TB diagnostics is an Xpert MTB/RIF test. It in allows to detect TB with the rifampicin resistance from sputum in just 2 hours after collection. The biggest advantage of the Xpert MTB/RIF test is simplicity in conduction of the test itself and the rapidity of testing which was described above [33]. Moreover, this test showed its high sensitivity and specificity which was approved in recent studies: with the using of Xpert MTB/RIF test in 98.2\% (551 out of 561) of the sputum smear-positive TB cases and $72.5 \%$ (124 out of 171) of those with sputum smear-negative TB the TB was detected. In comparison with the DST, the Xpert MTB/ RIF assay correctly identified $97.6 \% \quad(200$ out of 205) of patients harboring rifampicinresistant strains and 98.1\% (504 out of 514) of those with rifampicin-susceptible strains [34]. Based on this results the WHO recommends the using of Xpert MTB/RIF for patients with suspected multi-drug resistant TB. This test was also recommended for using among patients with the suspected pulmonary TB with negative smear test. However, despite all the advantages of this method it should be used mostly for the people with suspected multidrug resistant TB forms, because its PPV is significantly affected in the group of patients in which the rifampicin prevealance rate falls below $5 \%$. In such case the phenotypic DST or LPA should be used [31]

\section{CLINICAL FEATURES OF PRIMARY}

Primary TB usually presents as a low-grade, ebrile illness lasting for two to three weeks with 'other' symptoms in less than $25 \%$ of patients. Other symptoms include pleurisy pain, fatigue, cough, arthralgia and pharyngitis. Examination reveals chest pain to palpation and features of an effusion.

\section{Typical Clinical Outcome}

The initial focus of infection becomes infiltrated with lymphocytes and activated macrophages. Ipsilateral hilar lymph nodes (site of antigen presentation) become enlarged. The combination of a periphera lung infection and enlarged lymph nodes is then called a Ghon complex. Successful containment of the infection may result in residual scarring of the primary lung infection site and calcification after some years (Ghon focus). The clinical diagnosis of primary infection is therefore made on the basis of an altered skin test and radiological indenopathy or middle and

\section{Diagnostic algorithms of pulmonary $T B$} Smear-positive pulmonary TB

Each individual with suspected TB should go through the medical examination. The basic test is a chest X-ray which then should be followed with rational diagnostic procedures based on its results. The next step should be the multiple sputum examination (at least two specimens should be examined) [36]. After the sputum test the NAA tests could be performed in order to distinguish $M$ tuberculosis and nontuberculosis mycobacteria (NTM) in a 


\section{Diagnostic Algorithm for Pulmonary TB}

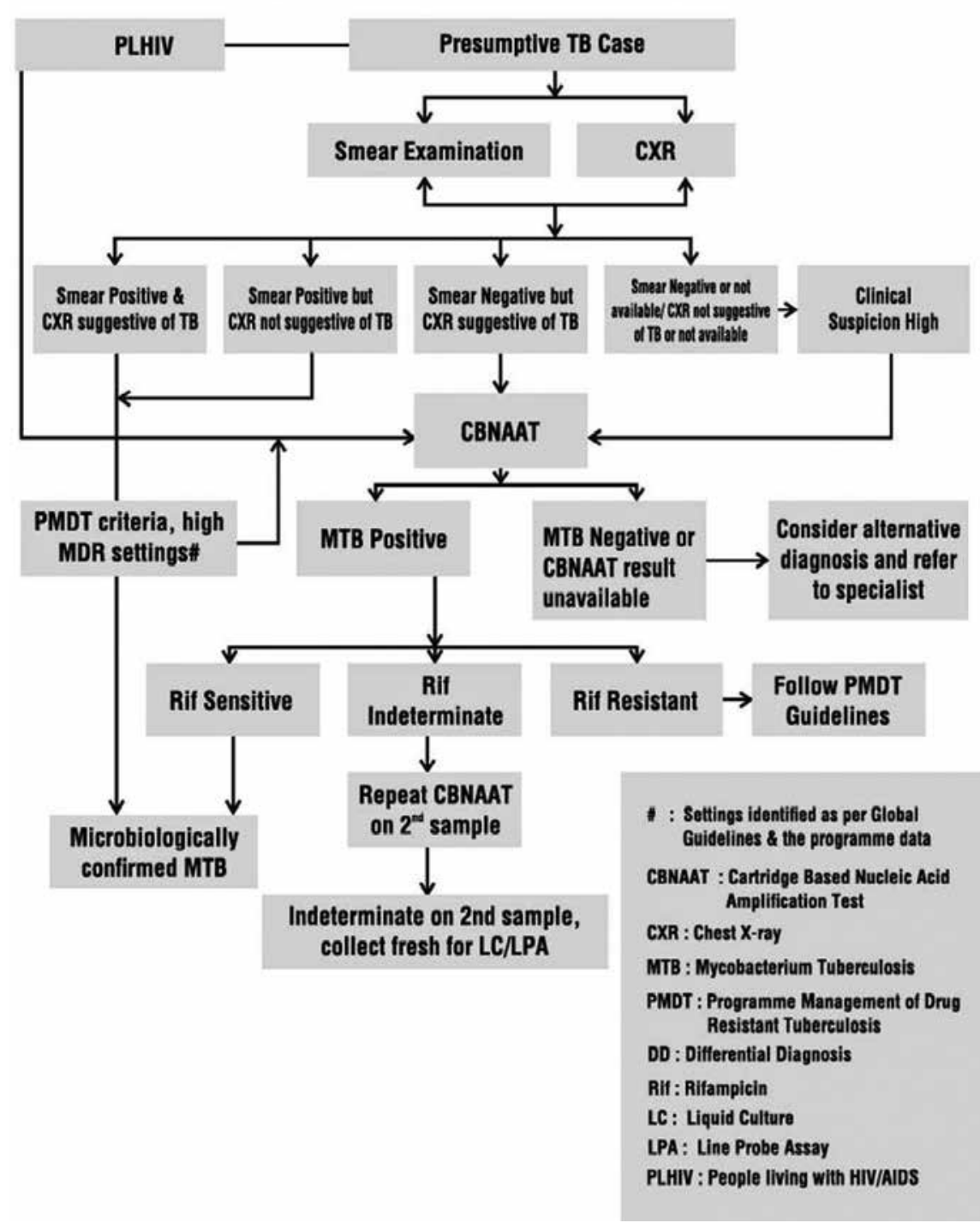

sputum smear-positive person. If the results of both AFB and NAA tests are positive, it allows to confirm the TB diagnosis.

Despite all the new possibilities and conveniences which new diagnostic methods could provide the culture and conventional DST still remains as ones of the most necessary tests in confirmation of $\mathrm{Tb}$ diagnosis $[25,37,38,39]$.

\section{TREATMENT}

Early diagnosis and effective anti-tuberculous chemotherapy will result in a cure in most cases.

Standard short-course chemotherapy for drug-susceptible tuberculosis consists of four drugs (rifampicin, isoniazid, pyrazinamide and ethambutol-intense four-drug regime) for two months followed by two drugs (rifampicin and isoniazidconsolidation two-c two weeks of appropriate therapy, although dead mycobacteria may still be visible in the sputum for some weeks following initiation of therapy. Fixed drug combinations allow simpler prescribing and may increase patient compliance but are less flexible should side effects become a problem $[32,35]$.

\section{PREVENTION AND INDICATION}

The preventive treatment or treatment of latent tuberculosis infection (LTBI) is recommended for individuals who are infected with Mycobacterium tuberculosis and those who have an increased risk of reactivation. Considering the statistical risk of reactivation, the indications for preventive therapy are, by decreasing order of magnitude: • HIV-positive patients with a positive tuberculin skin test (or positive interferon- $\gamma$ test) • Recent contact with smear-positive cases of TB with tuberculin conversion (or positive interferon- $\gamma$ test) $\cdot$ Children with a positive tuberculin skin est (or positive interferon- $\gamma$ test) • Subjects (ith untreated pulmonary TB - Subjects with natural, viral or drug-induced immune depression and with positive tuberculin skin test (or positive interferon- $\gamma$ test) $[28,37]$.

\section{CONCLUSION}

Tuberculosis infection and disease are not the same. One-third of the world's population is infected, but the majority do not experience disease. Transmission of tuberculosis is person-to person by droplet infection. Patients with cough and cavitary disease are the most infectious. Close contacts and people with immunodeficiency are at greatest risk of infection. In Primary disease, Mycobacterium infection. In Primary disease, Mycobacterium tuberculosis infects an immunologically naive
host and immune responses occur for the first time. Often this is asymptomatic but progressive disease can result, particularly in children or adults with immunodeficiency. Post-primary disease is when $\mathrm{M}$. tuberculosis causes symptoms either by reactivation of primary infection or reinfection with a new strain. Symptoms are due to the combined effect of pathogen replication and host esponse. The diagnosis of tuberculosis begins with clinical suspicion. The classic symptoms, however, can be mimicked by other conditions, including malignancies and systemic and pulmonary infections. Further HIV co-infection has changed the clinical presentation of tuberculosis requiring an increased clinical suspicion to engage modern diagnostic techniques with a wider range of patients. tuberculosis can be identified in the sputum of an organism or other clinical samples, Radiology and other tests are supportive but do not make the diagnosis. the dis caust Untreated, tubercusis progresses to cause lung damage and death, but effective cos patients. Also, early diagnosis and appropriate treatment are essential for good outcomes.

\section{REFERENCES}

. World Health Organsiation: TB Factsheet. 2011 Pub Who.

2. Onyebujoh $\mathrm{P}$, Rodriguez $\mathrm{W}$, and Mwaba $\mathrm{P}$ Priorities in tuberculosis research. Lancet 2006;367:940-2.

3. Raviglione MC. The global plan to stop TB, 2006-2015, Int J Tuberc Lung Dis 2006;10:238-9.

4. World Health Organization. Global tuberculosis report 2014 [Internet] Geneva: World Health Organization; 2014. [cited 2015 Mar 1].

5. World Health Organization. Early detection Wo tuberculosis: an overview of approachguidelines and tools es, guidelines and tools [Internet] Geneva:
World Health Organization; 2011.

6. The radiographic features of pulmonary tuberculosis. Woodring $\mathrm{JH}$, Vandiviere $\mathrm{HM}$ Fried AM, Dillon ML, Williams TD, Melvin IG AJR Am J Roentgenol. 1986 Mar 146(3):497-506.

. Radiologic features of pulmonary tuberculosis: an assessmen of 188 cases. Krysl J Korzeniewska-Kosela M, Müller NL, FitzGerald JM Can Assoc Radiol J. 1994 Apr; 45(2):101

8. Community-acquired pneumonia and tuberculosis: differential diagnosis and the 
use of fluoroquinolones. Grossman RF Hsueh PR, Gillespie SH, Blasi F Int J Infect Dis. 2014 Jan; 180):14-21.

9. Is the delay in diagnosis of pulmonary tuberculosis related to exposure to fluoroquinolones or any antibiotic? Wang $M_{1}$ Fitzgerald JM, Richardson K, Marra CA Cook VJ, Hajek J, Elwood RK, Bowie WR Marra F Int J Tuberc Lung Dis. 2011 Augi 15(8):1062-8.

10. Nardell EA. Catching droplet nuclei: toward a better understanding of tuberculosis transmission. Am J Respir Crit Care Med 2004;169:553-4.

11. Cegielski JP and McMurray DN. The relationship between malnutrition and tuberculosis: evidence from studies in humans and experimental animals. Int J Tuberc Lung Dis 2004;8:286-98.

12. Deiss RG, Rodwell TC, and Garfein RS. Tuberculosis and illicit drug use: review and update. Clin Infect Dis 2009;48(1):72

13. Syed Suleiman SA, Ishaq Aweis DM, Mohamed AJ, RazakMuttalif $A$, and Moussa MA. Role of diabetes in the prognosis and therapeutic outcome of tuberculosis. Int Endocrinol 2012;2012:645362.

14. Tang NL, Fan HP, Chang KC, et al. Genetic association between a chemokine gene CXCL-10 (IP-10, interferon gamma inducible protein 10) and susceptibility to tuberculosis. Clin Chim Acta 2009;406:98- 102.

15. Lienhardt C, Bennett S, Del Prete G, et al. Investigation of environmental and host-related risk factors for tuberculosis in Africa. I. Methodological aspects of a combined design. Am J Epidemiol 2002;155:1066-73.

34 16. Keane J, Gershon S, Wise RP, et al. Tuberculosis associated with infliximab, a tumo necrosis factor alpha-neutralizing agent. $N$ Engl J Med 2001;345:1098-104.

17. Joint Committee for the Revision of Korean Guidelines for Tuberculosis, Korea Centers for Disease Control and Prevention. Korean guidelines for tuberculosis. 2nd ed. Seoul and Cheongwon: Joint Committee for the Revision of Korean Guidelines for Tuberculosis, Korea Centers for Disease Control and Prevention; 2014

18. Adult-onset pulmonary tuberculosis: findings on chest radiographs and $\mathrm{CT}$ scans. Lee KS, Song KS, Lim TH, Kim PN, Kim IY, Lee BH AJR Am J Roentgenol. 1993 Apr 160(4):753-8.

19. Pulmonary tuberculosis: CT findings--early active disease and sequential change with antituberculous therapy. Im JG, Itoh $\mathrm{H}_{\text {, }}$ Shim YS, Lee JH, Ahn J, Han MC, Noma S Radiology. 1993 Mar; 186(3):653-60.

20. International standards for tuberculosis care 3rd ed [Internet] The Hague: TB Care 1; 2014.

21. World Health Organization. Same-day diagnosis of tuberculosis by microscopy: WHO policy statement [Internet] Geneva: World Health Organization; 2011. [cited 2015 Mar 1]. Available from:

22. Diagnostic accuracy of same-day microscopy versus standard microscopy for pulmonary tuberculosis: a systematic review and meta-analysis. Davis JL, Cattamanchi A, Cuevas LE, Hopewell PC, Steingart KR Lancet Infect Dis. 2013 Feb; 13(2):147-54.

23. Fluorescence versus conventional sputum smear microscopy for tuberculosis: a systematic review. Steingart $K R$, Henry $M, N g$ V, Hopewell PC, Ramsay A, Cunningham J, Urbanczik R, Perkins M, Aziz MA, Pai M Lancet Infect Dis. 2006 Sep; 6(9):570-81.

24. Current evidence on diagnostic accuracy of commercially based nucleic acid amplification tests for the diagnosis of pulmonary tuberculosis. Greco S, Girardi E, Navarra A, Saltini C Thorax. 2006 Sep; 61(9):783-90.

25. Commercial nucleic-acid amplification tests for diagnosis of pulmonary tuberculosis in respiratory specimens: meta-analysis and meta-regression. Ling DI, Flores LL, Riley LW, Pai M PLoS One. 2008 Feb 6; 3(2):e1536.

26. Updated guidelines for the use of nucleic acid amplification tests in the diagnosis of tuberculosis. Centers for Disease Control and Prevention (CDC). MMWR Morb Mortal Wkly Rep. 2009 Jan 16; 58(1):7-10.

27. Diagnostic Standards and Classification of Tuberculosis in Adults and Children. This official statement of the American Thoracic Society and the Centers for Disease Control and Prevention was adopted by the ATS Board of Directors, July 1999. This statement was endorsed by the Council of statement was endorsed by the Council of the Infectious Disease Society of AmeriCa, September 1999. Am J Respir Crit

28. World Health Organization. Molecular line probe assays for rapid screening of patients at risk of multidrug-resistant tuberculosis (MDR-TB): policy statement [Internet] Geneva: World Health Organization 2008. [cited 2015 Mar 1].

29. Mechanisms of drug resistance in Mycobacterium tuberculosis. Zhang $Y$, Yew WW Int J Tuberc Lung Dis. 2009 Nov; 13(11):1320-30.

30. World Health Organization. Molecular line probe assays for rapid screening of patients at risk of multidrug-resistant tuberculosis (MDR-TB): policy statement [Internet] Geneva: World Health Organization 2008. [cited 2015 Mar 1].

31. World Health Organization. Automated real-time nucleic acid amplification technology for rapid and simultaneous detection of tuberculosis and rifampicin resistance: Xpert MTB/RIF assay for the diagnosis of pulmonary and extrapulmonary TB in adults and children: policy update adults and chi Health Organization; 2013. [cited 2015 Mar 1].

32. Rapid molecular TB diagnosis: evidence, policy making and global implementation of Xpert MTB/RIF. Weyer K, Mirzayev F, Migliori GB, Van Gemert W, D'Ambrosio $L$, Zignol M, Floyd $K$, Centis R, Cirillo DM, Tortoli E, Gilpin C, de Dieu Iragena J, Falzon D, Raviglione M Eur Respir J. 2013 Jul; 42(1):252-71.

33. World Health Organization. Policy statement: automated real-time nucleic acid amplification technology for rapid and simultaneous detection of tuberculosis and rifampicin resistance: Xpert MTB/RIF system [Internet] Geneva: World Health Organization; 2011. [cited 2015 Mar 1]

34. Rapid molecular detection of tuberculosis and rifampin resistance. Boehme $\mathrm{CC}_{\text {, }}$ Nabeta P, Hillemann D, Nicol MP, Shenai S, Krapp F, Allen J, Tahirli R, Blakemore R, Rustomjee R, Milovic A, Jones M, O'Brien SM, Persing DH, Ruesch-Gerdes S, GotuzSO, E, Rodrigues $C$, Alland D, Perkins Gotuz zo E, Rodrigues C, Alland D, Perkins MD
N Engl J Med. 2010 Sep 9; 363(11):1005-15.

35. Xpert $\circledast$ MTB/RIF assay for pulmonary tuberculosis and rifampicin resistance in adults. Steingart $K R$, Schiller I, Horne DJ, Pai M, Boehme CC, Dendukuri N Cochrane Database Syst Rev. 2014 Jan 21; (1):CD009593

36. Usefulness of sputum induction with hypertonic saline in a real clinical practice for bacteriological yields of active pulmonary tuberculosis, Seong GM, Lee $J$, $J H_{1}$ Kim JH, Kim M Tuberc Respir Dis (Seoul). 2014 Apr; 76(4):163-8.

37. International standards for tuberculosis care. Wkly Epidemiol Rec. 2006 Feb 3; 81(5):43-7.

38. Differences in clinical presentation among persons with pulmonary tuberculosis: a comparison of documented and undocumented foreign-born versus US-born persons. Achkar JM, Sherpa T, Cohen HW Holzman RS Clin Infect Dis. 2008 Nov 15; 47(10):1277-83.

39. Low sensitivity of a whole-blood interferon-gamma release assay for detection of active tuberculosis. Dewan PK, Grinsdale J, Kawamura LM Clin Infect Dis. 2007 Jan 1; 44(1):69-73.

Corresponding author: Andriy Yu. Horoshchak, a.horoshchak@kmu.edu.ua

Manuscript was recieved on 25 October; accepted for publication on 26 December.

CITE AS: PETRO V. KUZYK ET AL. CHALENGES IN MORPHOLOGICAL DIAGNOSIS TUBERCULOSIS

BIOMEDICAL UPDATE, ISSUE 1, 202 